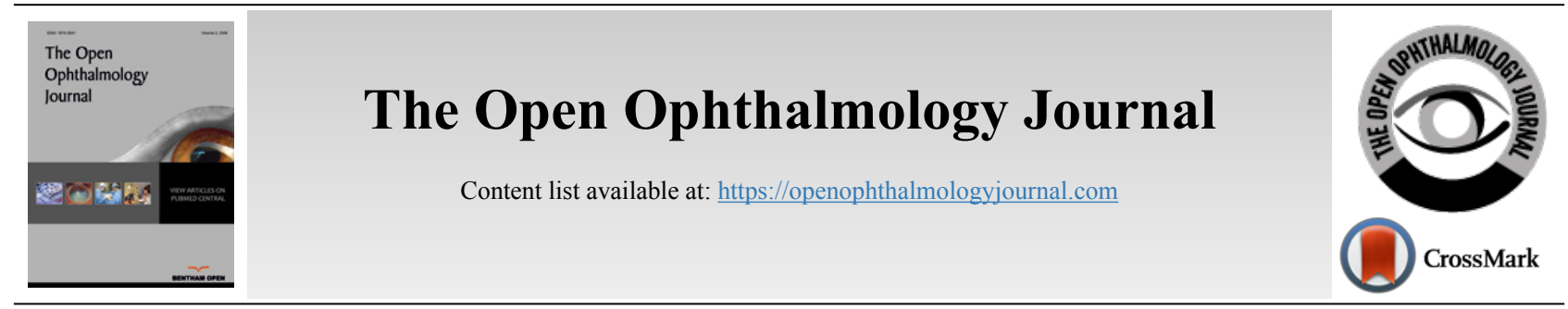

RESEARCH ARTICLE

\title{
Publication Rates of Registered Clinical Trials in Diabetic Macular Edema
}

\author{
Patrick J. Hughes ${ }^{1, *}$, Richard N. Polo ${ }^{1}$, Howard F. Fine ${ }^{1,2}$ and Jonathan L. Prenner ${ }^{1,2}$ \\ ${ }^{\prime}$ Department of Ophthalmology, Rutgers Robert Wood Johnson Medical School, New Brunswick, NJ, USA \\ 2Department of Ophthalmology, NJRetina, New Brunswick, NJ, USA
}

\begin{abstract}
:
Introduction:

To determine the publication rate of registered clinical trials evaluating therapeutic approaches for diabetic macular edema (DME).

Methods:

Using the search terms "Diabetic Macular Edema," the National Institute of Health's ClinicalTrials.gov online registry was searched to identify all clinical trials concerning DME. Non-interventional trials, terminated trials, trials not concerning DME, and those not completed between 2005 and 2015 were excluded. Publication status of each trial was determined using searches of the study title, keywords, author(s), and NCT number on PubMed.gov and Google Scholar.

Results:

A total of two hundred and forty-seven studies were identified in the primary analysis. Of the 97 trials meeting inclusion criteria, $60(61.9 \%)$ were published. Late-phase trials were published at higher rate $(74.5 \%$ [35/47]) than early phase trials $(50 \%[25 / 50])(p=0.01)$. Trial location, date of completion, and industry involvement did not significantly affect the publication rate.

Conclusion:

Of the registered trials listed in the ClinicalTrials.gov registry investigating DME, $61.9 \%$ were published.
\end{abstract}

Keywords: Clinical trial, Diabetic macular edema, Diabetic retinopathy, Publication, Registry, Retina, Vascular endothelial growth factor.

\begin{tabular}{l|l|l|l} 
Article History & Received: February 28, 2020 & Revised: October 26, 2020 & Accepted: November 17, 2020
\end{tabular}

\section{INTRODUCTION}

Diabetic Retinopathy (DR) remains a leading cause of blindness among working-age adults [1]. Diabetic Macular Edema (DME) is the most common form of treatable vision loss in the diabetic population, and because of this, it has garnered significant attention from clinical investigators. The advent of novel therapeutic interventions, both regional depot steroids and anti-Vascular Endothelial Growth Factor (VEGF) agents, has led to the ability to manage DME and conserve functional vision, even in poorly controlled patients [2].

The emergence of these novel therapeutic approaches led investigators to perform a large number of clinical trials to test

\footnotetext{
* Address correspondence to this author at the Department of Ophthalmology, Rutgers Robert Wood Johnson Medical School, New Brunswick, NJ 10 Plum Street, Suite 600, New Brunswick, NJ, USA; Tel: 609-240-6424; Fax: 732-220-1603; E-mail: pjh127@rwjms.rutgers.edu
}

varying innovations and clinical paradigms in an effort to refine therapeutic regimens in the treatment of DME. Clinical trials represent a crucial means of investigating the safety, efficacy, and use of novel therapeutic interventions for myriad retinal pathologies. However, the results of many clinical trials are not archived in a published, searchable manner. The absence of these critical outcome measures has the potential of leading investigators to subsequently research strategies or test novel therapeutic assets that have previously been shown to be ineffective.

We have previously analyzed the publication rate for clinical trials concerning exudative AMD spanning the period from 1998 to 2009, noting that nearly half of all trials conducted during this time frame failed to produce results published in the peer-reviewed literature [3]. While all clinical trials are required to be registered and publicly listed on ClinicalTrials.gov as of 1999, such requirements for 
publication of study outcomes were not required to be listed until the year 2017, at which time these regulations were expanded to require the recording of "clinical trials of drug products (including biological products) and device products that are not approved, licensed, or cleared by FDA" [4, 5]. In this investigation, we analyzed the publication rate of registered clinical trials in DME over a ten-year period and compared these results with those of our earlier study of AMD.

\section{MATERIALS AND METHODS}

All trials included for consideration in this study were identified by two independent researchers (P.H., R.P.) on the National Institute of Health's ClinicalTrials.gov using the search terms "diabetic macular edema." Non-interventional trials, terminated trials, trials not concerning DME, and those not completed between January 2005 and January 2015 were all excluded. This time frame was chosen to allow adequate time for publication following trial completion. Trials that did not specify a study phase ("Not Applicable") were also excluded. Within each study listing on the registry, the following data were extracted and recorded: official title, study type, study phase, actual study completion date, location(s), investigator(s), sponsor(s), NCT number, and the presence or absence of study results within the online registry. Studies for which results had been submitted but not yet posted were categorized as production of results for our records distinct from the publication of those results in the medical literature.

The publication status of each trial was determined using a series of relevant search terms, including the study title and investigator(s) in both PubMed.gov and Google Scholar. For searches using PubMed.gov, two methods of searching were employed. The first method entailed a search of the official study title as listed in the registry, with the addition of the primary investigator's surname for ambiguous results or an absence of results on the initial search. The second method involved searching for the study using the ClinicalTrials.gov Identifier, or NCT number. Regardless of the findings using PubMed.gov, Google Scholar was also employed to assess publication status by searching for the official study title with the addition of the primary investigator's surname for ambiguous results or absence of results on the initial search. For studies that failed to initially produce results on PubMed.gov but were located using Google Scholar, the exact publication title found on Google Scholar was then searched on PubMed.gov. A trial was deemed published if the study was identified on either PubMed.gov, Google Scholar, or both. A Chi-square analysis was used to compare categorical variables affecting publication rate using a significance level of $p=0.05$.

\section{RESULTS}

The primary ClinicalTrials.gov registry search yielded 247 trials concerning DME. Of these, $150(60.7 \%)$ were excluded because they were non-interventional, terminated, not exclusively investigating DME, not completed between January 2005 and January 2015, or did not specify the study phase. The subsequent $97(39.3 \%)$ remaining trials were included in the final analysis. Of these 97 trials, 60 (61.9\%) met the criteria for publication in the peer-reviewed medical literature. Forty-one (42.3\%) trials included study results under the appropriate header on the registry. Google Scholar searches captured the highest number of published trials at 57 (95\% of published), with PubMed.gov capturing 48 ( $80 \%$ of published). Searches of the NCT number captured the fewest publications at 40 (66.7\% of published).

In addition to analyzing the rate of publication for trials investigating DME, factors affecting the likelihood of publication were also investigated. Trials were categorized as domestic (exclusively within the United States) or international. Domestic trials were published at a rate of 55.3\% (21 of 38), whereas the publication rate for international trials amounted to $66.1 \%$ (39 of 59). Trial location did not significantly affect the rate of publication for DME trials ( $p=$ 0.28).

The phase of a clinical trial was also evaluated as a factor that might influence the publication rate in DME trials. Studies in phases I, I/II, and II were considered "early-phase" while those in phases II/III, III, and IV were categorized as "latephase." Late-phase studies demonstrated a significantly higher publication rate relative to early-phase studies at $74.5 \%$ (35 of $47)$ and $50 \%$ (25 of 50), respectively ( $p=0.01)$.

A third factor considered in publication rate was the role of industry sponsorship or involvement in the trial. Trials without industry involvement or sponsorship were published at a slightly higher rate $(68.3 \%$ [28/41]) than those receiving industry support $(57.1 \%[32 / 56])$. However, this difference was not statistically significant $(p=0.26)$.

Finally, the year of trial completion was considered as a factor affecting the publication rate (Fig. 1). Trials completed between the year 2005 and 2009 were published at a rate of $63.3 \%$ ( 19 of 30 ), while trials completed between the period spanning 2010 to 2015 were published at a rate of $61.2 \%$ (41 of $67)$. This difference was not statistically significant $(p=0.84)$.

Our prior study of the publication rate of clinical trials in AMD between January 1998 and January 2009 demonstrated a publication rate of $54 \%$ ( 35 of 64 ). This current analysis of the publication rate in clinical trials in DME between January 2005 and January 2015 yielded a publication rate of $61.9 \%$ (60 of 97). There was no significant difference in publication rate between registered clinical trials in AMD and DME $(p=0.37)$. 


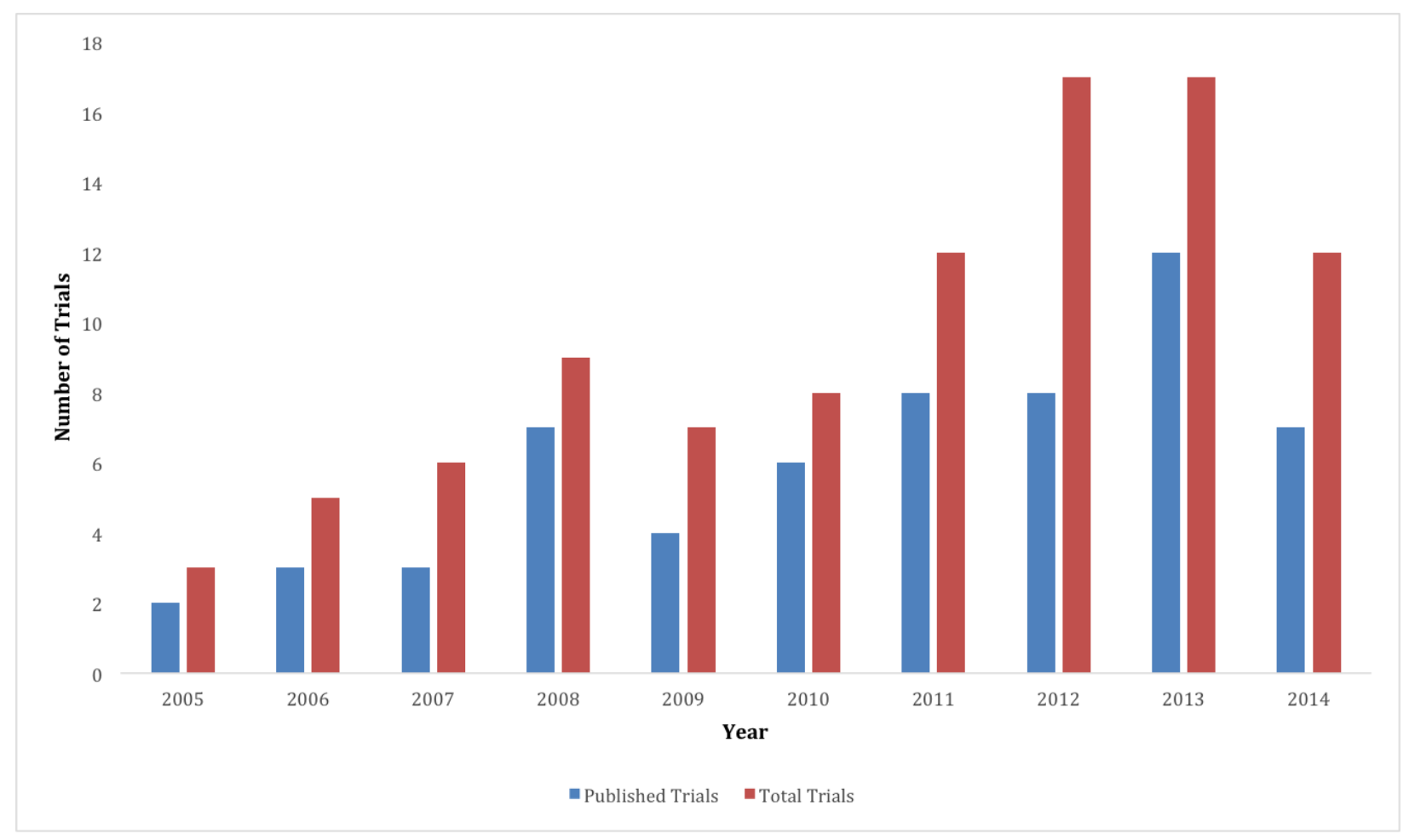

Fig. (1). Quantity of trials per year of completion.

This graphic shows the distribution and quantity of published and unpublished trials by year of completion.

\section{DISCUSSION}

The results produced by clinical trials represent critical information that guides the development and advancement of novel therapeutic interventions for DME. This investigation of the publication rate of completed DME trials reveals that nearly one-half fail to produce published and searchable results in the medical literature. Consequently, it is difficult to quantify the volume of information about innovative treatments and treatment strategies for DME that have been tested but remain unknown to the scientific community.

This discrepancy could lead to the duplication of experimental interventions by multiple investigators resulting in a misallocation of resources and exposure of patients to potential harms from interventions already known to be ineffective from prior unpublished trials. Given the weight these trials carry in dictating clinical practice, it should stand to reason that both providers and patients may benefit from the publication of all results, thereby ensuring that clinical decision making is informed by all available information, both positive and negative. Since 2017, trial results have been required to be listed in an abbreviated format on the ClinicalTrials.gov website. However, limited trial information is included in these datasets relative to those in a scientific publication, and data from previous trials are not captured in this fashion.

Of particular interest to our group is the comparison between publication rates for trials investigating DME and AMD, as elucidated in a prior study, and to trend any variations in this metric in the interval following our first analysis. At the time of its completion, our analysis demonstrated that the publication rate for registered clinical trials in AMD was 54\% and noted that late-phase trials were published at a significantly higher rate than early-phase trials. The location, date of study initiation, and extent of industry involvement had no effect on publication rate. By comparison, our investigation of registered clinical trials in DME revealed a publication rate of $61.9 \%$, also demonstrating a significantly higher percentage of publication in late-phase trials compared to early-phase trials while study location, date of study completion, and industry involvement had no effect. Thus, the higher likelihood of publication for late-phase trials relative to early-phase trials has persisted. However, there was no statistically significant difference in the publication rate of trials concerning DME and AMD.

The reasons that many clinical trial results remain unpublished are myriad. Investigators encounter significant resistance when attempting to publish trials that do not have positive results in the medical literature, as there is no "Journal of Negative Results". Our study did not differentiate between studies that produced positive or negative results due to the subjective nature of that classification. However, trials with negative results are much more challenging to publish and likely influence the overall publication rate. Investigators and industry sponsors may lose their enthusiasm for a particular innovation or clinical paradigm after it has been proven ineffective and then have little interest in publishing those data. Since our last publication outlining this trend in AMD, it appears as though little has changed with regard to the publication rates produced by clinical trials appearing in the medical literature, albeit for a different ophthalmic pathology, but within a more recent time frame. 
While a higher volume of trials in DME has been completed since 2012, driven by investigation of anti-VEGF agents, the proportion of those trials attaining publication has remained relatively unchanged (Fig. 1). This persistence highlights the stagnation in the number of clinical trials whose results are available to be viewed by the physician-scientist community. The unavailability of this data, particularly for early-phase trials assessing the safety and efficacy of novel interventions and treatment strategies, permits the repetition of trials that have already been shown to be ineffective or potentially harmful to patients. Creating a resource that allows investigators to log their progress for innovative treatments or alterations to commonplace practices may facilitate more practical use of time and resources such that duplicate studies are not unnecessarily repeated.

This study has several limitations. First, by confining our time frame of trial completion, it is plausible that these results have failed to capture additional published trials, particularly in more recent years as the use of the anti-VEGF agents has gained acceptance. We also chose not to incorporate the results of two trials added to the registry while data collection was in progress. Consequently, this analysis may underestimate the rate of publication for registered clinical trials in DME. Additionally, the use of only two search sources to determine the publication status of a given trial may not identify results attaining publication in lesser-known journals, introducing a bias in which studies appearing in more reputable journals are easier to locate across a variety of search platforms.

\section{CONCLUSION}

In summary, $61.9 \%$ of registered clinical trials addressing DME over a ten-year time period published results in the peerreviewed medical literature. This result is consistent with those of our earlier study concerning publication rates in clinical trials for AMD, highlighting the continued absence of critical information garnered from these trials in sources available for review by physician-scientists. A consolidation or centralization of the information attained from all trials regarding a specific topic may enhance the safety of clinical research and inform the design of future studies.

\section{ETHICS APPROVAL AND CONSENT TO PARTI- CIPATE}

Not applicable.

\section{HUMAN AND ANIMAL RIGHTS}

No animals/humans were used for studies that are the basis of this research.

\section{CONSENT FOR PUBLICATION}

Not applicable.

\section{AVAILABILITY OF DATA AND MATERIALS}

Not applicable.

FUNDING

None.

\section{CONFLICT OF INTEREST}

The authors declare no conflict of interest, financial or otherwise.

\section{ACKNOWLEDGEMENTS}

Declared none.

\section{REFERENCES}

[1] Klein BE. Overview of epidemiologic studies of diabetic retinopathy. Ophthalmic Epidemiol 2007; 14(4): 179-83.

[http://dx.doi.org/10.1080/09286580701396720] [PMID: 17896294]

[2] Virgili G, Parravano M, Evans JR, Gordon I, Lucenteforte E. Antivascular endothelial growth factor for diabetic macular oedema: A network meta-analysis. Cochrane Database of Systematic Reviews 2018; 10(6) Available from: https://www.cochranelibrary.com/cdsr/ doi/10.1002/14651858.CD007419.pub6/full [http://dx.doi.org/10.1002/14651858.CD007419.pub6]

[3] Prenner JL, Driscoll SJ, Fine HF, Salz DA, Roth DB. Publication rates of registered clinical trials in macular degeneration. Retina 2011; 31(2): 401-4.

[http://dx.doi.org/10.1097/IAE.0b013e3181 eef2ad] [PMID: 21221049]

[4] Laine C, Horton R, DeAngelis CD, et al. Clinical trial registration-looking back and moving ahead. N Engl J Med 2007; 356(26): 2734-6. [http://dx.doi.org/10.1056/NEJMe078110] [PMID: 17548427]

[5] Federal Register. Office of the Federal Register. National Archives and Records Administration 2016; 81(183): 64759-5168. 